\title{
Experimental signatures of Anderson localization of light in three dimensions
}

\author{
C. M. AEGERTER*, M. STÖRZER, \\ W. BÜHRER, S. FIEBIG and G. MARET \\ Fachbereich Physik, Universität Konstanz, Box M621, \\ D-78457 Konstanz, Germany
}

\begin{abstract}
The observation of Anderson localization of light has long been hindered by the lack of clear-cut experimental signatures. Static transmission measurements for instance would show an exponential decrease of intensity, which cannot be distinguished from absorption. Here we present time-of-flight measurements of single photons in three-dimensional samples. At long times, localization leads to a less than exponential decrease of transmission, which is observed for very turbid samples. While absorption cannot account for such a non-exponential decay, it is still important to determine the absorption length independently. This can be achieved from reducing the index mismatch of the scatterers and performing similar time-of-flight measurements. Such a decrease of the scattering power of the particles also shows that the only sample property leading to non-classical diffusion is indeed the turbidity $1 / k l^{*}$ as predicted theoretically.
\end{abstract}

\section{Introduction}

For the last two decades, there were many attempts to observe signatures of Anderson localization [1] of visible light [2,3]. Until recently, progress in this field was hampered by the fact that clear-cut experimental signatures for localization were not commonly known. For instance in some experiments, the focus was on a determination of static transmission [4], which due to localization of photons should be suppressed exponentially with sample thickness $L$ [2]. However, only in the absence of absorption such an exponential decrease in transmission is a qualitative change from a non-localizing sample [5, 6]. In samples close to the localization transition the mean free path $l^{*}$ is extremely small and therefore transmission paths are necessarily long, leading to a non-negligible absorption in real experiments [7]. Therefore, an exponential decrease in transmission is only quantitatively different in localizing and non-localizing samples. Thus, static transmission measurements are solely able to indicate the presence of localization if the absorption properties of the

\footnotetext{
*Corresponding author. Email: christof.aegerter@uni-konstanz.de
} 
samples are measured independently and the decrease in transmission is faster than that expected from absorption alone [8]. In fact in the presence of an additional characterization of localization, as discussed below, it should be possible to describe static transmission measurements without any adjustable parameters. In that case, static transmission measurements are highly useful, as they present an independent and quantitative self-consistency check whether the observed phenomenon obeys the predictions of localization [8].

On the other hand, the dependence of the diffusion coefficient on increasing disorder was studied [9]. There it was found that when the scatterer size is changed in order to reduce $l^{*}$, the diffusion coefficient is strongly reduced compared to its expected value. In that case, however, the particle size becomes comparable to the wavelength of light, such that resonance scattering [10] may become important. This point was made quantitative by van Albada et al. [11]. They found that the increased dwell time due to resonant scattering does indeed lead to a reduction in the transport velocity and thus the diffusion coefficient, $D \propto v_{\mathrm{T}} l^{*}$. As we will discuss below, however, the Mie resonances [12] in the scattering cross-section leading to the reduction in transport velocity and the resonances leading to increased scattering and thus localization are not exactly the same, such that the two effects can be separated experimentally [13].

These problems with identifying experimental signatures for Anderson localization imply that a determination of both the absorption length and the transport velocity is necessary. This is done in the study reported here.

Considering the basic predictions of Anderson localization theory, i.e. the renormalization of the diffusion coefficient with $L$ [14], time-of-flight measurements have been proposed as ideal candidates for signatures of localization [15-17]. This is because effectively in time-resolved measurements the spread of the photon cloud within the sample can be obtained. Thus at long times, the transmitted intensity is simply given by $\exp \left(-\pi^{2}\left\langle r^{2}\right\rangle / L^{2}\right)$. For a diffusive process with a mean square displacement $\left\langle r^{2}\right\rangle=D t$, this leads to a simple exponential decay of transmission. In the presence of absorption, this exponential decay is enhanced by the inverse absorption time $1 / \tau_{\mathrm{abs}}$, however, it stays purely exponential [17]. Localization in contrast would lead to a saturation in the growth of $\left\langle r^{2}\right\rangle$ and hence to a slower-thanexponential decay of the time-resolved transmission at long times [18]. Furthermore, the absorption length can still be determined from the additional exponential decay and the diffusion coefficient at short times is also measured. Combining time-of-flight measurements with coherent backscattering experiments furthermore, yields a direct determination of the transport velocity $v_{\mathrm{T}}$ [13]. Here, we will discuss experiments to this effect on strongly scattering $\mathrm{TiO}_{2}$ powders with particles sizes in the range of $220-540 \mathrm{~nm}$. Performing these experiments for samples with various values of $\mathrm{kl}^{*}$ then also gives information on details of the phase transition to localization.

\section{Experimental setup}

The time-of-flight measurements are performed using a single photon counting technique. Pulses of a width of $\sim 20$ ps are passed through the sample and the time 
of flight of photons is measured from the time difference of the signal from a photomultiplier behind the sample and that of a reference diode in front of the sample $[8,18]$. In these measurements it is important that only a fraction of a photon (statistically) passes through the sample in order to avoid timing bias towards shorter transmission times. A histogram of many such delay times gives the time-of-flight distribution of the photons through the multiple scattering sample. In order to correct for the effects of (strongly suppressed) after-pulses as well as of indiscriminate noise, our data are deconvoluted with the input pulse, which is measured before and after the measurement. Comparing both reference measurements, we are able to identify laser drifts, e.g. in intensity and pulse width, easily. In this way, we obtain the path length distribution of photons inside the sample, which can be compared with theoretical predictions that are analytically available in the case of diffusion. The determination of $D$ and $\tau_{\text {abs }}$ is reasonably independent, as absorption is only important in the decay of the intensity at long times, whereas the diffusion coefficient determines the time delay of the photons first leaving the sample.

For the determination of the transport velocity, we independently measure the turbidity $\left(k l^{*}\right)^{-1}$ from the width of the coherent backscattering cone [19, 20]. Following the theoretical treatment by Akkermans et al. [21], the shape of the cone can be fitted with the single parameter of $k l^{*}$, which essentially describes the width. We use a special setup designed to measure the backscattered light over a very wide angular range of $-60^{\circ}$ to $85^{\circ}$ with high accuracy [22]. This allows a proper determination of the width, as the incoherent background following a Lambert-Beer dependence [23] can be determined even for very wide cones. Due to the strong scattering of the $\mathrm{TiO}_{2}$ particles, the samples have a reasonably high refractive index, which leads to internal reflections of the backscattered light at the surface. This effectively reduces the width of the backscattering cone [24], which has to be corrected using the value of the effective refractive index of the sample. The correction can be calculated using the energy coherent potential approximation [25] given the size of the particles and their refractive index.

The samples themselves consist of ground $\mathrm{TiO}_{2}$ in its rutile structure, which is commercially available from DuPont and Aldrich [18]. The size distribution of the particles is determined using scanning electron microscopy, which usually yields a polydispersity of $20 \%$. The electron micrographs also show that the ground particles are non-spherical [18]. For the different samples, the average particle size ranges from 220 to $540 \mathrm{~nm}$.

\section{Results}

Previous experiments have shown that $\mathrm{TiO}_{2}$ particles with an average diameter of $250 \mathrm{~nm}$ show strong deviations from classical diffusion at packing fractions around $50 \%$ [18]. These time-of-flight distributions can be described by a phenomenological theory based on a time-dependent diffusion coefficient [26, 27], as demanded by localization theory [14]. Representative data and a fit with this phenomenological theory are shown in figure 1. As can be seen, at long times the decrease in transmission is markedly slower than the exponential (indicated by the 


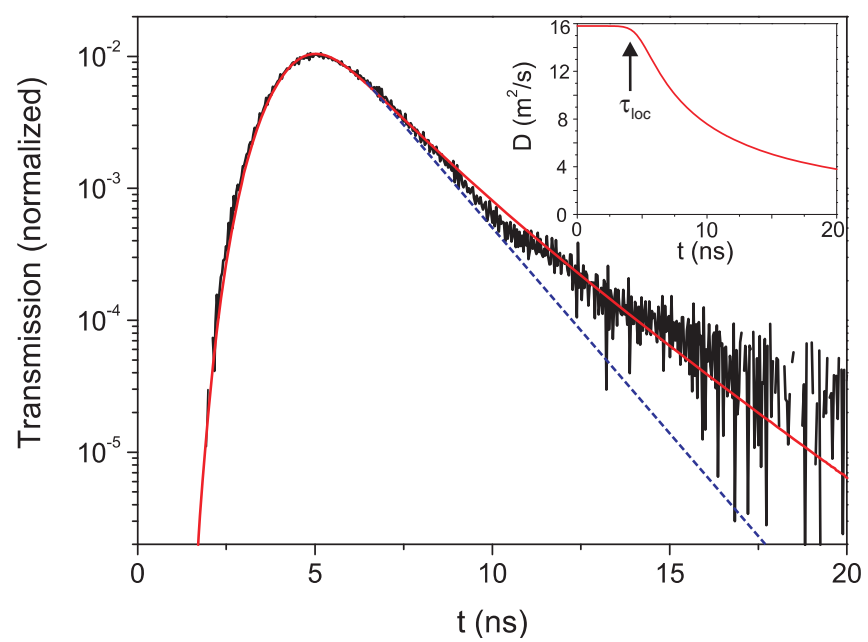

Figure 1. Time-of-flight measurements from a $\mathrm{TiO}_{2}$ sample with a value of $k l^{*}$ of 2.5 . At long times, there are clear deviations from an exponential decay as predicted by diffusion with absorption (dashed blue line). The data can however be described by a temporally varying diffusion coefficient (see inset), which is predicted for Anderson localization. This results in the full red line in the plot. (The colour version of this figure is included in the online version of the journal.)

dashed line). This shows that a process different from diffusion or absorption is at work. To be more quantitative, a modified diffusion theory [8] can be used to fit the data. The main input for this description is the time dependence of the diffusion coefficient shown in the inset of figure 1 . Here, $D$ is constant up to a certain time, $\tau_{\text {loc }}$ and then decreases as $1 / t$ at long times. This behaviour of $D(t)$ has also been found in numerical simulations of a self-attracting random walk [28]. Furthermore, it can be translated into a behaviour of $\left\langle r^{2}\right\rangle$, which corresponds to the intuitive description of localization discussed above of a diffusive photon cloud that saturates at a length scale corresponding to the localization length. The fit to this description shown in the full line of figure 1 yields the following values for the absorption time, $\tau_{\text {abs }}=2.0(1) \mathrm{ns}$, the diffusion coefficient, $D=15(1) \mathrm{m}^{2} \mathrm{~s}^{-1}$ and the localization length, $L_{\mathrm{loc}}=240(10) \mu \mathrm{m}$. Together with a determination of $k l^{*}=2.5(3)$, one can also directly calculate the transport velocity $v_{\mathrm{T}}=0.63 \mathrm{c}$. This is consistent with the speed of light divided by the Garnett refractive index [29], which indicates that resonant scattering is not important in this particular sample. Below we will discuss the dependence of $v_{\mathrm{T}}$ on the different samples, which shows that localizing samples can indeed also show resonant scattering.

A systematic study of the dependence of these deviations on the control parameter $k l^{*}$ can then give information about the transition to Anderson localization of light [8]. For instance, we have determined the critical value of $k l^{*}$ at which the phase transition to localization takes place as $k l_{c}^{*}=4.2(2)$. This is somewhat higher than the prediction by Ioffe and Regel [30] demanding unity for the 
critical value. Note, however, that the Ioffe-Regel argument is based on dimensional analysis and cannot predict constants of order one. Given this critical value for $k l^{*}$ it is then also possible to determine the critical exponent of the localization length. This turns out to be $v \simeq 0.45$ [8], which indicates that first-order perturbation theory [3] cannot be used to describe the transition. In that case the scaling theory of localization [14] predicts a value of one in disagreement with the experiment. The general prediction of scaling theory of $v<1$, however, is fulfilled and in good agreement with the expectation of $1 / 2$ for a standard order parameter [31]. Numerical investigations on quasi-periodic lattices have, however, found values of $v=1.5$ [32], which are not compatible with these experiments and may be due to the non-random nature of the lattice used in these investigations.

In the following, we will be concerned with experimental checks of the above assertion that absorption and decreased transport velocity can be properly distinguished from localization.

\subsection{Absorption}

In order to determine the influence of absorption on our signal we investigate the most localizing sample in more detail. Apart from localization, there are other effects which might possibly give rise to a non-exponential tail in the time-of-flight measurements. These include inhomogeneous layering, background illumination [18] as well as induced fluorescence [33], which could give rise to higher intensities at long times. An ideal way to exclude most of these experimental artefacts is to show that only the turbidity $k l^{*}$ influences the appearance of a non-exponential tail and no other sample properties are of influence. In order to do this, we have index-matched the most localizing sample by filling the voids between the grains with dodecane. Dodecane has a refractive index of $\sim 1.45$ and thus strongly reduces the scattering power of the particles, which increases $k l^{*}$ by roughly a factor of 10 . Therefore, by keeping the sample as well as the illumination intensity the same in the indexmatched experiments, one can rule out most of the above artefacts as the source of the non-exponential tail. To show that inhomogeneous layering does not cause the tail, we have previously shown that the illumination direction does not change the results [18]. In addition, an index-matched experiment can be used as another independent determination of the absorption length. Dodecane might lead to a somewhat increased absorption, such that this experiment will yield an upper limit.

Figure 2 shows the results of a time-of-flight measurement of an index matched sample. As can be seen, the data are well described by diffusive transport (full line), which yields $D=290(10) \mathrm{m}^{2} \mathrm{~s}^{-1}$ and $\tau_{\mathrm{abs}}=1.9(1) \mathrm{ns}$. This is in good agreement with the absorption length obtained from the pure sample using the phenomenological localization theory discussed above. Therefore, the absorption can be directly measured in time-of-flight data and does not give rise to the non-exponential tail observed in strongly turbid samples. The deviations at very short times visible in figure 2 are due to an increased rise time of the pulse in this experiment, which is not accounted for in the deconvolution.

Thus, having two independent determinations of the absorption length, we can perform the self-consistency check discussed above and measure the thickness 


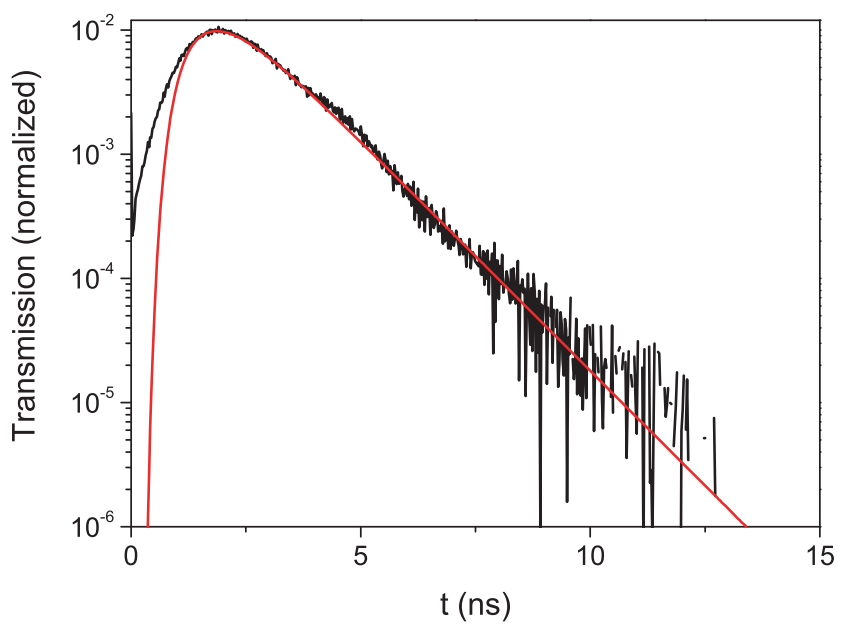

Figure 2. Time-of-flight distribution from a sample of $\mathrm{TiO}_{2}$ particles in dodecane. The $\mathrm{TiO}_{2}$ particles used in this sample are the same as those giving rise to the non-classical distribution shown in figure 1. As can be seen, a reduction in refractive index contrast leads to a classical time-of-flight distribution and an increase of $k l^{*}$. Furthermore, the addition of dodecane can only increase the absorption but should not greatly change the absorption time $\tau_{\text {abs }}$. Thus, the results from these measurements can be used as an upper limit to the absorption of the localizing sample. (The colour version of this figure is included in the online version of the journal.)

dependence of static transmission. The result of this is shown in figure 3 , where a clear exponential suppression of transmission can be seen in the data. This is obviously in disagreement with the expectation from pure diffusion which is indicated in the green dotted line in the figure. However, we have shown that samples with values of $k l^{*}$ above the transition also show exponentially decreasing static transmission [7]. In that case however, the absorption length from time-offlight measurements can describe the data. This is shown in figure 3 by the dashed and dashed-dotted lines. For the localizing sample studied here, these are still in stark disagreement with the data, such that we again have to conclude that diffusion and absorption cannot describe the data. The difference between the dashed and the dashed-dotted lines is that the dashed line is obtained from the determination of the absorption length using index-matching as shown in figure 2 above and thus presents an upper limit of the absorption present in the sample. The dashed-dotted line is obtained directly from the localization fit shown in figure 1 above. In order to properly describe the data, we have to also include the exponential suppression of the intensity due to localization, which is given by the localization length and which we have determined from the fit in figure 1 above. This yields the full red line in figure 3, which perfectly describes the data. The shaded area between the lines indicates the uncertainty in the quantities determined in the fit, i.e. the absorption length and the localization length. Note that therefore there is no adjustable parameter in the description of the static transmission which is in perfect agreement with the data over 12 orders of magnitude. We therefore conclude that absorption does not influence 


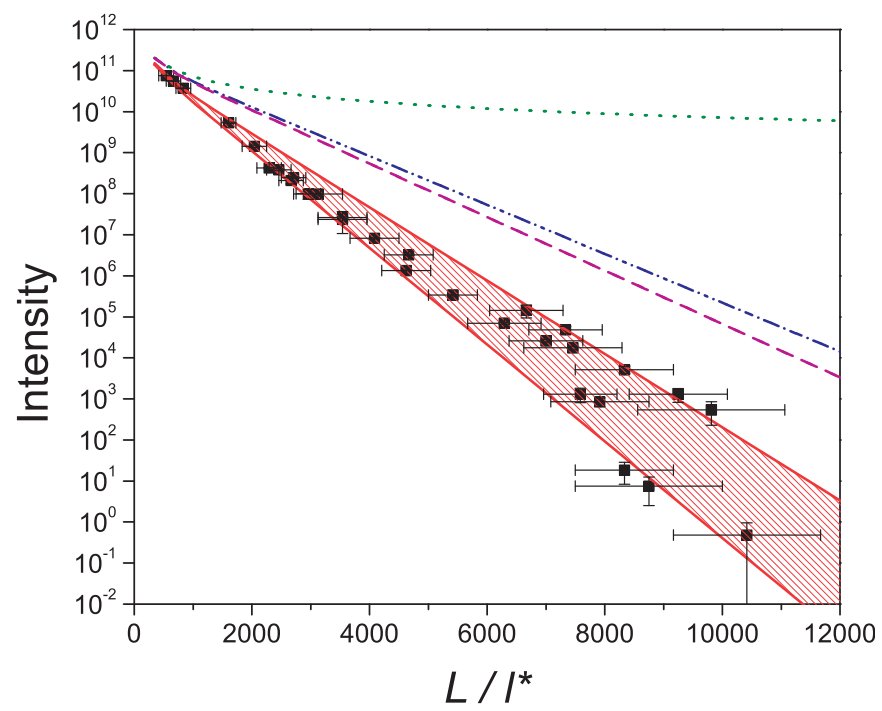

Figure 3. Static transmission measurements as a function of thickness through the most localizing sample. The dotted green line corresponds to the expectation of pure diffusion theory. The dashed and dashed-dotted lines describe diffusion in the presence of absorption. As is expected and also found experimentally, the static transmission decreases exponentially. However, the absorption length is too large to describe the data properly. Here, the dasheddotted line corresponds to the value obtained from the localizing sample itself, whereas the dashed line corresponds to that inferred from the index-matched sample. Only the full red line, which also takes into account the decrease of intensity due to localization, describes the experiments over the whole range and without any adjustable parameters. (The colour version of this figure is included in the online version of the journal.)

our findings and the non-exponential tail in the time-resolved transmission data is in good agreement with the suppression of static transmission.

\subsection{Transport velocity}

The second property we will look at more closely is the transport speed. As discussed above, a slowing down in transport as expected by localization can also be obtained from a reduction in transport velocity due to resonant scattering. In the reduction of $k l^{*}$ one uses the property of the Mie resonances [12] to increase the scattering power of the particles and thus reduce $l^{*}$. Similarly, the resonances are at the origin of the increased dwell time and thus the reduction in transport velocity is due to Mie resonances [11]. Therefore, it might be thought that resonant scattering and turbidity always go together and one could therefore expect a systematic dependence of $v_{\mathrm{T}}$ on $k l^{*}$. This is, however, not the case, as $l^{*}$ is not only influenced by the resonances in the form factor but also by those in the structure factor of the powder. This could already be guessed by the result above that the most localizing sample did in fact not show any significant reduction in transport velocity and could be described by the Garnett refractive index [29]. Systematically, this is shown in figure 4, where we show the dependence of both $v_{\mathrm{T}}$ (open circles) and $l^{*}$ (full squares) on the particle diameter 


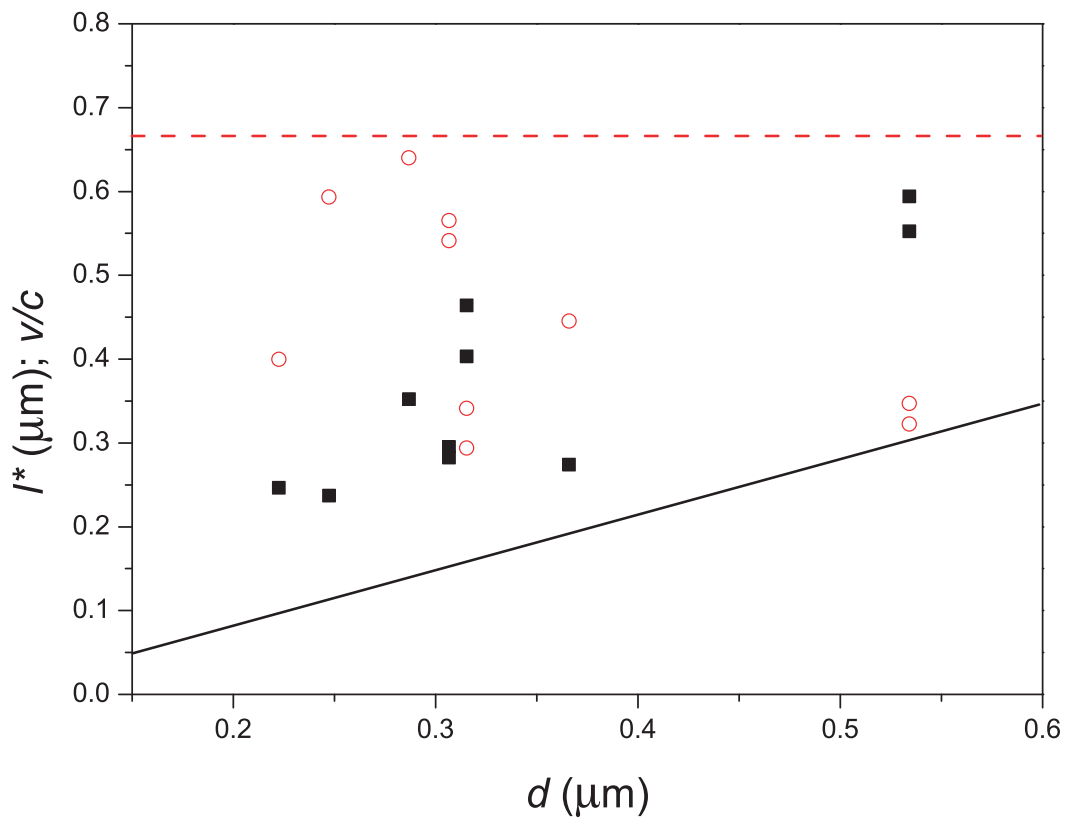

Figure 4. A comparison of the dependence of $l^{*}$ (full symbols) and $v_{\mathrm{T}}$ (open symbols) on the diameter of the scatterers. As can be seen, the resonances in the two properties do not match perfectly. This is due to the fact that the reduction in transport velocity is only due to resonances in the form factor, whereas the decrease in $l^{*}$ is due to a combination of the form and structure factors. The dashed line indicates the value of $v_{\mathrm{T}}$ obtained using the Garnett refractive index [29], while the full line corresponds to an estimation of $l^{*}$ assuming the scattering cross-section to be given by the size of the scatterer. (The colour version of this figure is included in the online version of the journal.)

in the samples. As can be seen, the resonances in both quantities, as given by the deviation from the classical expectations indicated by the solid and dashed lines, respectively, do not match up. For example at particle sizes around $300 \mathrm{~nm}$, there is increased resonance scattering as evidenced by a decrease in $v_{\mathrm{T}}$ but a decrease in scattering power as shown by an increase in $l^{*}$. This shows that effects of localization due to a minimization of $l^{*}$ can be well separated from those of resonance scattering.

Now we can also show that the non-exponential tail in the time-resolved transmission data is not due to a resonant scattering induced reduction in $v_{\mathrm{T}}$. As shown above, a systematic dependence on $k l^{*}$ as shown for the non-exponential tail $[8,18]$ cannot be obtained from a reduction in $v_{\mathrm{T}}$ alone. However, it might still be possible that the polydispersity of the samples leads to the presence of some slowed photons which would give rise to a non-exponential tail. In that case though, samples with a non-exponential tail should all show a mean $v_{\mathrm{T}}$ which is not reduced. Otherwise, particles in the tails of the size distribution would be non-resonant and thus would lead to an increased $v_{\mathrm{T}}$. This would then not lead to an upwards tail and thus could not be mistaken for localization. Figure 5 shows the transport velocity as a function of the localization length. Thus, if the non-exponential tail would be 


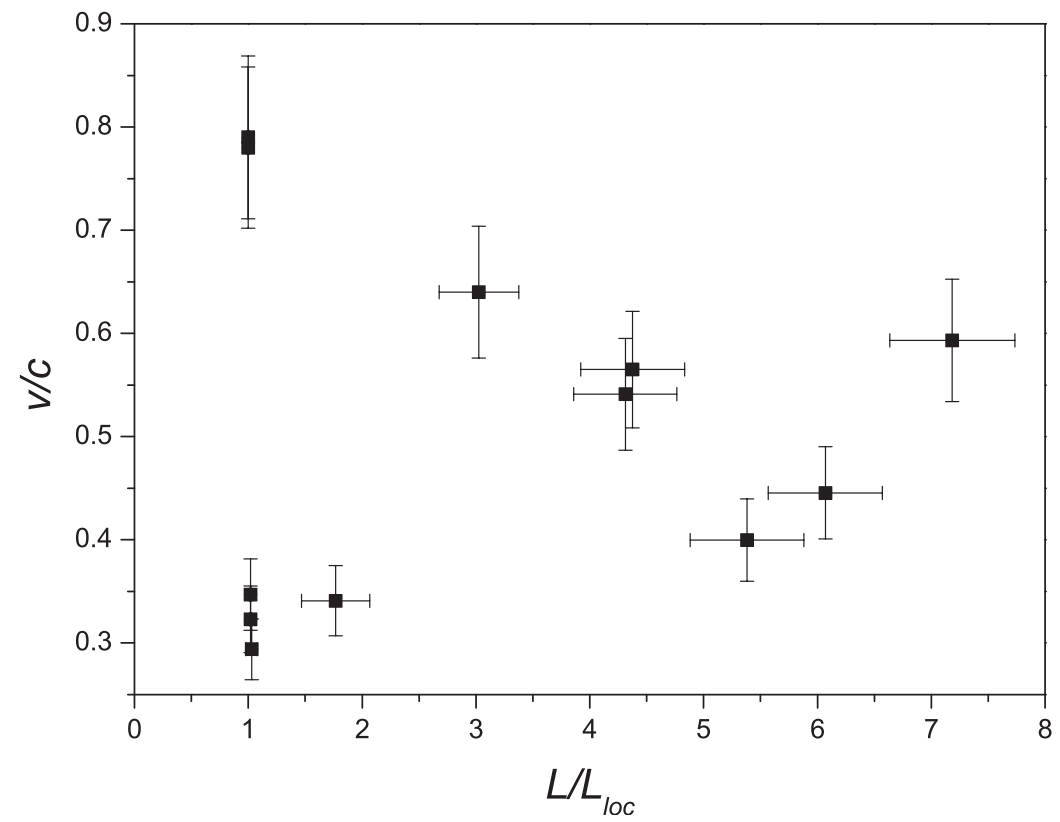

Figure 5. The transport velocity compared to the localization length as determined from time-of-flight measurements. As can be seen, the two measures do not correlate, such that localizing samples (with values of $L / L_{\mathrm{loc}}$ bigger than one) may have both high or low $v_{\mathrm{T}}$, similar to non-localizing samples (with $L=L_{\mathrm{loc}}$ ).

caused by the tails in the particle size distribution, we would expect the points on the right, where samples localize, to be higher. This is not the case and there are samples showing a non-exponential tail which do have strongly reduced transport velocities. Furthermore, the figure shows again that $v_{\mathrm{T}}$ and localization are not correlated. We find high and low values of $v_{\mathrm{T}}$ both for localizing and non-localizing samples.

Thus, we have shown that a reduction in $v_{\mathrm{T}}$ due to resonant scattering can be obtained from our samples, but that it is quite distinct from the effects of localization indicated by the non-exponential tail in time-of-flight measurements. Also a more subtle argument based on the polydispersity of the samples can be ruled out by the fact that resonance scattering can also be observed in localizing samples.

\section{Conclusions}

In conclusion, we have demonstrated that time-resolved transmission measurements of light through extremely turbid media show strong deviations from diffusive behaviour. These deviations are well described by the localization of photons to length scales of the localization length. Usual experimental difficulties, such as absorption and resonant scattering have been excluded as influencing our results. In fact we can quantitatively determine both the relevant quantities and use them in 
independent consistency checks showing that all of these results are in agreement with an interpretation of the data in terms of Anderson localization of light. Furthermore, using partial index-matching of the sample, we have shown that the only parameter influencing the appearance of non-diffusive transport is the turbidity $k l^{*}$. This shows that experimental artefacts such as fluorescence, inhomogeneous layering or background illumination can be ruled out as a source of the non-exponential tail in the time-of-flight measurements.

In the future, the time-of-flight data should also be described by a less phenomenological theory, such as that by Skipetrov and van Tiggelen [34], which explicitly describes open media. This also includes a quantitative measure of fluctuations, i.e. speckle intensity distributions close to the critical point.

\section{Acknowledgements}

This work was supported by the Deutsche Forschungsgemeinschaft, the International Research and Training Group 'Soft Condensed Matter of Model Systems' and the Center for Applied Photonics (CAP) at the University of Konstanz. We would also like to thank Peter Gross for help in the building of the backscattering cone setup. The samples used in this study were provided by DuPont, which is gratefully acknowledged.

\section{References}

[1] P.W. Anderson, Phys. Rev. 1091492 (1958)

[2] P.W. Anderson, Phil. Mag. B 52505 (1985).

[3] S. John, Phys. Rev. Lett. 532169 (1984).

[4] D.S. Wiersma, P. Bartolini, A. Lagendijk, et al., Nature (London) 390671 (1997).

[5] F. Scheffold, R. Lenke, R. Tweer, et al., Nature (London) 398206 (1999); see also D.S. Wiersma, J. Gómez Rivas, P. Bartolini, et al., reply ibid. 398207 (1999).

[6] A.A. Chabanov, M. Stoytchev and A.Z. Genack, Nature (London) 404850 (2000).

[7] C.M. Aegerter, M. Störzer, S. Fiebig, et al., J. Opt. Soc. Am. 24 A23 (2007).

[8] C.M. Aegerter, M. Störzer and G. Maret, Europhys. Lett. 75562 (2006).

[9] J.M. Drake and A.Z. Genack, Phys. Rev. Lett. 63259 (1989).

[10] E.P. Wigner, Phys. Rev. 98145 (1955).

[11] M.P. van Albada, B.A. van Tiggelen, A. Lagendijk, et al., Phys. Rev. Lett. 663132 (1991).

[12] G. Mie, Ann. Phys. 25377 (1908).

[13] M. Störzer, C.M. Aegerter and G. Maret, Phys. Rev. E 73 065602(R) (2006).

[14] E. Abrahams, P.W. Anderson, D. Licciardello, et al., Phys. Rev. Lett. 42673 (1979).

[15] G.H. Watson, P.A. Fleury and S.L. McCall, Phys. Rev. Lett. 58945 (1987).

[16] P.M. Johnson, A. Imhof, B.P.J. Bret, et al., Phys. Rev. E 68016604 (2003).

[17] A.A. Chabanov, Z.Q. Zhang and A.Z. Genack, Phys. Rev. Lett. 90203903 (2003).

[18] M. Störzer, P. Gross, C.M. Aegerter, et al., Phys. Rev. Lett. 96063904 (2006).

[19] M.P. van Albada and A. Lagendijk, Phys. Rev. Lett 552692 (1985).

[20] P.E Wolf and G. Maret, Phys. Rev. Lett 552696 (1985).

[21] E. Akkermans, P.E. Wolf and R. Maynard, Phys. Rev. Lett. 561471 (1986). 
[22] P. Gross, Coherent backscattering close to the transition to strong localization of light, Diploma thesis, University of Konstanz (2005); P. Gross, M. Störzer, S. Fiebig, et al., Rev. Sci. Instrum. 78033105 (2007).

[23] M. Born and E. Wolf, Principles of Optics, 6th ed. (Pergamon Press, Oxford, 1980).

[24] J.X. Zhu, D.J. Pine, and D.A. Weitz, Phys. Rev. A 443948 (1991).

[25] K. Busch and C.M. Soukoulis, Phys. Rev. B 54893 (1996).

[26] R. Berkovitz and M. Kaveh, J. Phys.: Condens. Matter 2307 (1990).

[27] R. Berkovitz and M. Kaveh, Phys. Rev. B 369322 (1987).

[28] R. Lenke, R. Tweer, and G. Maret, Eur. Phys. J. B 26235 (2002).

[29] J.C.M. Garnett, Phil. Trans. R. Soc. A 203385 (1904).

[30] A.F. Ioffe, and A.R. Regel, Progress in Semiconductors 4237 (1960).

[31] H.G. Schuster, Z. Phys. 3199 (1978).

[32] A. MacKinnon and B. Kramer, Phys. Rev. Lett. 471546 (1981); T. Rieth and M. Schreiber, Z. Phys. B 10499 (1997).

[33] D.S. Wiersma, private communication.

[34] S.E. Skipetrov and B.A. van Tiggelen, Phys. Rev. Lett. 96043902 (2006). 